\title{
Using of Renewable Energy in Green Building to Achieve Sustainability
}

\author{
Mohamed Gaber \\ Designer Engineer, Electrical \\ Consultant, Cairo, Egypt
}

\author{
Mohamed Nageh \\ Researcher Assistant, Ain Shams \\ Univ., Cairo, Egypt
}

\author{
Khaled Hossam \\ Telecomm. Engineer, Alcan \\ Network Com., Cairo, Egypt
}

\author{
Wagdy R. Anis \\ Professor, Faculty of Eng. Ain Shams Univ., Cairo,
} Egypt

\author{
Ismail M. Hafez \\ Professor, Faculty of Eng. Ain Shams Univ., Cairo, \\ Egypt
}

\begin{abstract}
The world population is the total number of humans currently living. As of August 2016, it was estimated at 7.4 billion. This over population comes with limit of fossil fuel energy resources in our world. Wide focus has been on the negative environmental effects of using fossil fuels. This problem leads us to increase our gradual dependence on the renewable energy resources to overcome the huge demand of energy for the coming generations. Besides, continuous decrease of the $\mathrm{PV}$ module cost, it is interesting to consider the generation of electricity from the PV system. Moreover, the use of renewable energy resources, instead of, fossil fuels will be a good solution for the control of the environmental, social and economic problems of our communities. In this paper, a residential building (Villa) in Dubai, UAE, has been taken based on solar energy as a part of its electricity consumption. The result shows that the energy production is about $300 \mathrm{~kW}$ per day. For each similar residential building, which will provide 591 \$ per month for the country in the production of energy, where we able to apply the concept of sustainability in the field of energy, provide as much 295.5 liter per month from fossil fuels consumed to produce electricity. That's return on the environment in a positive way, by reducing carbon emissions $\left(\mathrm{Co}_{2}\right)$, which leads to reduce the effect of the global warming problem. Also, we provide a source of energy for the future generations. However the cost per produced $\mathrm{kWh}$ is less in case of using the fossil fuels in the electricity generation, this is our challenge to achieve the concept of sustainability. Furthermore, in this work a system design and cost analysis are considered.
\end{abstract}

\section{Keywords}

Photovoltaic (PV) System, On Grid, Renewable Energy, Green Building, Sustainability, Economical Analysis, Dubai, UAE.

\section{INTRODUCTION}

The U.S. Energy Information Administration's recently released International Energy Outlook 2016 (IEO2016) projects that world energy consumption will grow by $48 \%$ between 2012 and 2040. Most of this growth will come from countries that are not in the Organization for Economic Cooperation and Development (OECD), including countries where demand is driven by strong economic growth, particularly in Asia. Non-OECD Asia, including China and
India, accounts for more than half of the world's total increase in energy consumption over the projection period.

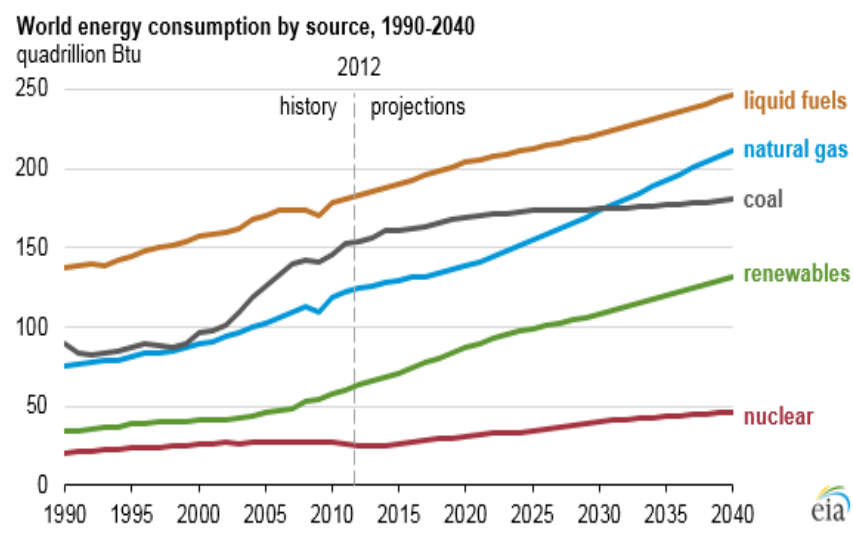

Fig 1: World energy consumption by source

Concerns about energy security, effects of fossil fuel emissions on the environment, and sustained, long-term high world oil prices support expanded use of non-fossil renewable energy sources and nuclear power. Renewable and nuclear power are the world's fastest-growing energy sources over the projection period. Renewable energy increases by an average $2.6 \%$ per year through 2040; nuclear power increases by $2.3 \%$ per year [1].

\subsection{Pros and Cons of Fossil Fuels}

1.1.1 Pros of fossil fuels [2]

1. Available in plenty.

2. Easier to find.

3. Extremely efficient.

4. Easier to transport.

5. Generate thousands of jobs.

6. Easy set up.

\subsubsection{Cons of fossil fuels [2]}

1. Environmental degradation.

2. Need truckloads of reserves.

3. Public health issues.

4. Finite energy source.

5. Oil spill. 
6. Rising cost.

7. Health of coal-mining workers.

\subsection{Pros and Cons of Nuclear Power}

\subsubsection{Pros of nuclear power [3]}

1. The easy availability of the materials used.

2. Easily transported.

3. Do not need a large area.

4. Nor does cause the emission of carbon dioxide $\left(\mathrm{Co}_{2}\right)$ and sulfur dioxide $\left(\mathrm{So}_{2}\right)$.

\subsubsection{Cons of nuclear power [4]}

Nuclear disasters destabilized the world:

1. The partial meltdown of a reactor for nuclear Sns Switzerland.

2. Three Mile Island - Pennsylvania.

3. Fire and Windscale station - Britain.

4. Fukushima - Japan.

5. Chornobyl disaster - Ukraine.

\subsection{Pros and Cons of Renewable Energy}

\subsubsection{Pros of renewable energy [5]}

1. Stable energy prices.

2. Continual source of energy.

3. Reliability.

4. Low greenhouse gas emissions.

5. Large scale job creation.

6. Low cost operation.

7. Micro-station options possible.

\subsubsection{Cons of renewable energy [5]}

1. High development cost.

2. Vulnerable public health issues.

3. Unable to produce in large quantities.

4. Not available in all areas.

5. Large areas required.

\subsection{What is Sustainability?}

Sustainability means meeting our own needs without Compromising the ability of future generations to meet their own needs. In addition to natural resources, we also need social and economic resources. Sustainability is not just environmentalism. Embedded in most definitions of sustainability, we also find concerns for social equity and economic development.

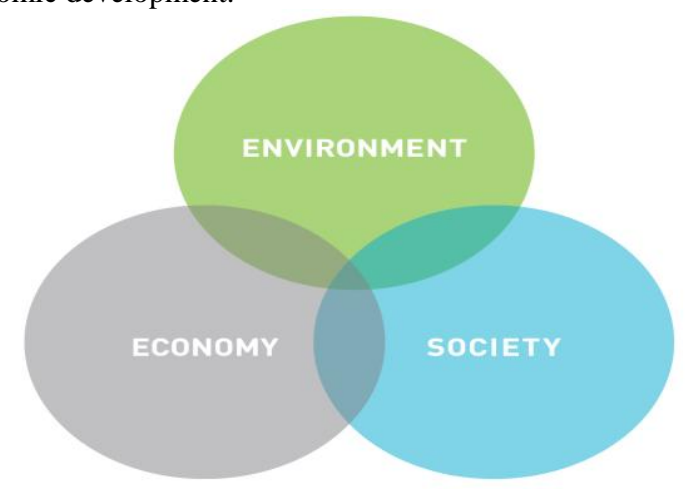

Fig 2: Three pillars of sustainability

\subsubsection{Environmental sustainability}

Ecological integrity is maintained, all of the earth's environmental systems are kept in balance while natural resources within them are consumed by humans at a rate where they are able to replenish themselves.

\subsubsection{Economic sustainability}

Human communities across the globe are able to maintain their independence and have access to the resources that they require, financial and other, to meet their needs. Economic systems are intact and activities are available to everyone, such as secure sources of livelihood.

\subsubsection{Social sustainability}

Universal human rights and basic necessities are attainable by all people, who have access to enough resources in order to keep their families and communities healthy and secure. Healthy communities have just leaders who ensure personal, labour and cultural rights are respected and all people are protected from discrimination [6].

\section{DESIGN}

This section discuss some aspects that need attention in the Arabic region, as it is characterized by the tremendous amount of resources, and feature geographic location, so that region has the great luck of solar radiation yearly, and that has not been exploited so far. The UAE is one of the world's highest rates of energy consumption per person, and reported per capita consumption between $20-30 \mathrm{kWh}$ of electricity a day, with the global rates ranging between 7-15 kWh.

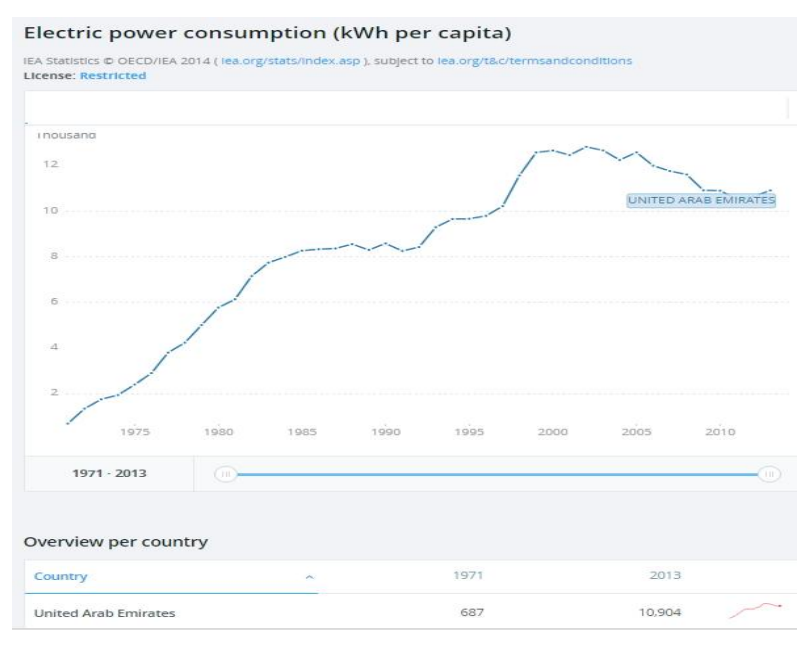

Fig 3: Electric power consumption [7]

Renewable energy plays a crucial role in the UAE's economic growth \& diversification plans. The UAE aims to bolster its regional position as a role model for sustainable development. We take our top model for a residential Villa in Dubai, United Arab Emirates (UAE), for 10 things to know about the UAE's renewable energy landscape.

1. The UAE has consistently demonstrated its willingness to be a world-leading advocate of clean energy technology. The country is looking to increase its target for power generation from clean energy to $30 \%$ by 2030 .

2. The UAE leadership aims to enhance the country's leadership in renewable energy in order to create an innovation-drive environment that generates jobs opportunities. The UAE will create more than 90,000 jobs in the renewable energy sector by 2030 . 
3. In terms of investment, the UAE is becoming a role model in renewable energy. The Abu-Dhabi based company Masdar has invested $\$ 2.7$ billion into clean-energy projects over the past decade.

4. Gulf countries will save around $\$ 87$ billion, which is equal to 2.5 billion barrels of oil equivalent, from lower oil and natural gas consumption if they achieve goals for renewable use by 2030 according to the Abu Dhabi-based International Renewable Energy Agency. The UAE's savings from the renewable energy sources were between $\$ 1$ billion and $\$ 3.7$ billion in 2015 .

5. The UAE government has committed over $\$ 340$ million for sustainable efforts in developing countries. The Abu Dhabi Fund for Development has announced $\$ 46$ million in loans for 4 renewable energy projects in Africa and the Caribbean.

6. Abu Dhabi's renewable energy company Masdar was official host partner to the Solar Impulse 2 that became the first aircraft to travel the globe without using a drop of fuel. The aircraft has flown day and night using only solar power in order to create more awareness on clean technologies.

7. Abu Dhabi's Masdar City is one of the best examples of the UAE's innovative and forwardlooking strategies to boost its renewable energy sector. The world's most sustainable eco-project is set to be completed by 2030 . It is expected that 40,000 residents and 50,000 people working at the Masdar City by 2030.

8. Dubai aims to become the city with the lowest carbon footprint in the world. Dubai aims to generate 7 percent of its power from clean energy sources by 2020, 25 percent by 2030, and a whopping 75 percent by 2050 .

9. Abu Dhabi's Shams-1 is the largest renewable energy project in operation in the Middle East. Shams-1 is a $100 \mathrm{MW}$ development that is one of the world's largest concentrated solar power (CSP) projects.

10. Mohammed Bin Rashid Al Maktoum Solar Park, the world's biggest solar park, is being built in Dubai. The solar park in $\$ 14$ billion will eventually produce 5,000 megawatts, which is enough to power 800,000 homes, in 2030. Mohammed Bin Rashid Al Maktoum Solar Park will produce power for as little as \$2.99 US cents per kilowatt-hour [8].

\section{DESIGN PROCEDURES}

\subsection{Temperature and Solar Irradiance of Dubai}

Table 1 . Monthly daily average energy $\left(H_{B}\right)$ produced by 1 kWp on horizontal surface and ambient temperature (Ta) in degree

\begin{tabular}{|c|c|c|c|c|c|c|}
\hline Month & Jan & Feb & Mar & Apr & May & Jun \\
\hline $\begin{array}{c}\text { Energy } \\
\mathbf{k W h} / \mathbf{m}^{2} / \mathbf{d a} \\
\mathbf{y}\end{array}$ & 6.68 & 7.84 & 9.25 & 10.49 & 11.26 & 11.56 \\
\hline
\end{tabular}

\begin{tabular}{|c|c|c|c|c|c|c|}
\hline $\begin{array}{c}\text { Temp in } \\
\text { degree }\end{array}$ & 23 & 24 & 27 & 32 & 37 & 38 \\
\hline Month & Jul & Aug & Sep & Oct & Nov & Dec \\
\hline $\begin{array}{c}\text { Energy } \\
\mathbf{k W h} / \mathbf{m}^{2} / \mathbf{d a} \\
\mathbf{y}\end{array}$ & $\begin{array}{c}11.4 \\
3\end{array}$ & 10.84 & 9.73 & 8.3 & 6.98 & 6.33 \\
\hline $\begin{array}{c}\text { Temp in } \\
\text { degree }\end{array}$ & 40 & 41 & 38 & 35 & 31 & 26 \\
\hline
\end{tabular}

\subsection{Equations}

\subsubsection{Solar declination $(\boldsymbol{\delta})$ [9]:}

The earth's axis of rotation is tilted at $23.45^{\circ}$ with respect to its orbit plane about the sun. The days when axis is tilted exactly toward or away from the sun are 21 June and 21 December, called Summer and Winter solstices. On 21 March and 21 September, called Spring and Fall equinox, when the tilt of the earth's axis is neither along the orbit and neither away nor to sun, defined as:

$$
\delta=23.45 \sin \left[(284+n) \times \frac{360}{365}\right]
$$

Where that: $\mathrm{n}=$ day of the year $(1 \leq \mathrm{n} \leq 365)$.

\subsubsection{Extraterrestrial solar irradiance (Hext) [9]:}

The monthly average daily extraterrestrial solar insolation $\left(\mathrm{kWh} / \mathrm{m}^{2} /\right.$ day) on horizontal surface at the same latitude $\emptyset$ of the site under consideration. The extraterrestrial solar irradiance $\left(\mathrm{kW} / \mathrm{m}^{2}\right)$ on a surface normal to solar beams is constant and equal to solar constant Gsc $=1.35 \mathrm{~kW} / \mathrm{m}^{2}$. Note that all of solar radiation in the extraterrestrial space is only direct radiation and thus no diffused radiation exists, defined as:

$\mathrm{H}_{\mathrm{ext}}=\frac{24}{\pi} \mathrm{G}_{\mathrm{SC}}\left[\cos \delta \cos \varphi \sin \omega_{\mathrm{s}}+\omega_{\mathrm{s}} \sin \delta \sin \varphi\right]$

\subsubsection{Solar radiation on horizontal surface $\left(H_{B}\right)$ [9]:}

The monthly average daily solar radiation on a horizontal surface HB (kWh/m2/day), defined as:

$$
H_{B}=H_{D} \cos \theta_{Z}+H_{d}
$$

\subsubsection{Clearness index $\left(K_{T}\right)$ [9]:}

The ratio between the solar radiation on horizontal surface $\mathrm{H}_{B}$ $\left(\mathrm{kWh} / \mathrm{m}^{2} /\right.$ day $)$ and the monthly average daily extraterrestrial solar insolation Hext $\left(\mathrm{kWh} / \mathrm{m}^{2} /\right.$ day $)$ on a horizontal surface, defined as:

$$
\mathrm{K}_{\mathrm{T}}=\frac{\mathrm{H}_{\mathrm{B}}}{\mathrm{H}_{\mathrm{ext}}}
$$

\subsubsection{Direct radiation on horizontal surface $\left(H_{D}\right)$ [9]:}

The direct radiation energy on a horizontal surface $H_{D}$ $\left(\mathrm{kWh} / \mathrm{m}^{2} /\right.$ day $)$, defined as:

$$
H_{D}=\frac{24}{\pi} G_{n}\left[\cos \delta \cos \varphi \sin \omega_{s}+\omega_{s} \sin \varphi \sin \delta\right]
$$

3.2.6 Direct radiation on tilted surface $\left(H_{T}\right)$ [9]: The direct radiation energy on a tilted surface $\mathrm{H}_{T}$ 
$\left(\mathrm{kWh} / \mathrm{m}^{2} /\right.$ day $)$, defined as:

$$
\begin{aligned}
& H_{T}=H_{B}\left[1.13 K_{T} R_{b}+0.5(1+\cos \beta)\left(1-1.13 K_{T}\right)+\right. \\
& 0.5 \rho(1-\cos \beta)]
\end{aligned}
$$

\subsubsection{The ratio between direct radiation on tilted} and horizontal surface $\left(R_{b}\right)$ [9]:

The ratio between the global solar radiation on tilted surface $\mathrm{H}_{\mathrm{T}}\left(\mathrm{kWh} / \mathrm{m}^{2} /\right.$ day $)$ and the global solar radiation on horizontal surface $\mathrm{H}_{\mathrm{B}}\left(\mathrm{kWh} / \mathrm{m}^{2} /\right.$ day $)$, defined as:

$$
R_{b}=\frac{H_{T}}{H_{B}}
$$

\subsubsection{Instantaneous global solar irradiance on tilted surface $\left(G_{T}\right)$ [9]:}

The instantaneous global solar irradiance on tilted surface GT $\left(\mathrm{kW} / \mathrm{m}^{2}\right)$, defined as:

$$
G_{T}=\frac{\pi}{24} H_{T} \frac{\cos \omega-\cos \omega_{s}^{\prime \prime}}{\sin \omega_{s}^{\prime}-\omega_{s}^{\prime} \cos \omega_{s}{ }^{\prime \prime}}
$$

3.2.9 Current output from $P V$ array $\left(I_{A}\right)$ [10]: The current output from PV array IA (A), defined as:

$$
\mathrm{I}_{\mathrm{A}}=\mathrm{I}_{\mathrm{SC}} \times \mathrm{G}_{\mathrm{T}}-\left(\mathrm{I} \circ \times \mathrm{e}^{\mathrm{V} / \mathrm{V}_{\mathrm{t}}}\right)
$$

3.2.10 Power output from PV array $\left(P_{\text {out }}\right)$ [11]: The power output from $\mathrm{PV}$ array Pout $(\mathrm{W})$, defined as:

$$
P_{\text {out }}=V_{\text {out }} \times I_{\text {out }}
$$

\subsubsection{Maximum power output from $P V$ array}

$$
\left(P_{\max }\right)[11] \text { : }
$$

The maximum power output from PV array Pmax (W), defined as:

$$
P_{\text {max }}=V_{P \max } \times I_{P \max }
$$

\section{PV Module Characteristics Used in the Design:}

Our design is based on 200 watt peak power module under AM1conditions with the terrestrial solar radiation is $G_{O}=1$ $\left(\mathrm{kW} / \mathrm{m}^{2}\right)$ and the cell temperature is $\mathrm{T}_{\mathrm{C}}=25^{\mathrm{O}} \mathrm{C}$. Its parameters are the open-circuit voltage is $\mathrm{V}_{\mathrm{OC}}=33 \mathrm{~V}$, the maximum power voltage is $V_{P \max }=25 \mathrm{~V}$, the short-circuit current is $\mathrm{I}_{\mathrm{SC}}=8.27 \mathrm{~A}$ and the maximum power current is $\mathrm{I}_{\mathrm{Pmax}}=8$ A.

PV modules sizes used in the design: If 5 modules connected with each other in series will give energy equal to $1 \mathrm{~kW} / \mathrm{m}^{2}$ which required an area equal to $8 \mathrm{~m}^{2}$ for monocystallinze silicon or $10 \mathrm{~m}^{2}$ for polycrystalline silicon. The design is based on $90 \mathrm{~kW}_{\mathrm{P}}$ which required 450 modules each one is 200 $\mathrm{W}_{\mathrm{P}}$. Then 450 (modules) $* 8 \mathrm{~m}^{2} * 0.2 \mathrm{~W}$ (module) $=720 \mathrm{~m}^{2}$ as a total area required for PV modules.

The Inverters used in the design: Inverters capacity is 5 (inverters) $* 20 \mathrm{~kW}_{\mathrm{P}}=100 \mathrm{~kW}_{\mathrm{P}}$ to cover $\left(90 \mathrm{~kW}_{\mathrm{P}}\right)$ design. Inverters sizes are 5 (inverters) $* 0.25 \mathrm{~m}^{2}$ (area for each one) $=1.25 \mathrm{~m}^{2}+1.25 \mathrm{~m}^{2}$ (separate spaces) $=2.5 \mathrm{~m}^{2}$.
The batteries used in the design: Batteries capacity is 18 (batteries) $* 12 \mathrm{~V}=216 \mathrm{~V}$. Batteries stored in a battery bank, which required an area equal to $1.5 \mathrm{~m}^{2}$ and stored in strings. Each string battery consists of 2 batteries together $(2 * 12 \mathrm{~V}=$ $24 \mathrm{~V}$ ) which means 9 (battery strings) * $170 \mathrm{Ah}$ (for each string) $=1500 \mathrm{Ah}$

In case of with battery, a PV system feeds only lighting loads as a part of total loads of the villa. In addition to, the average of lighting loads per 10 hours (average of night's hours in the UAE) equal to $32 \mathrm{~kW}$ per 10 hours.

\subsection{Block Diagram}

\subsubsection{Overall block diagram of PV system design}

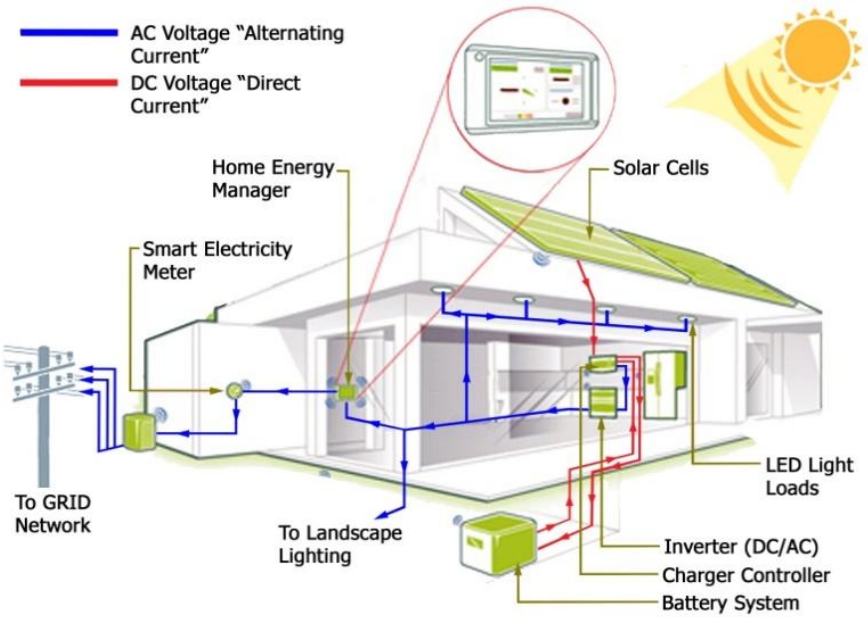

Fig 4: Overall block diagram connections inside villa

3.3.2 Current flow in components of PV system in case of without battery

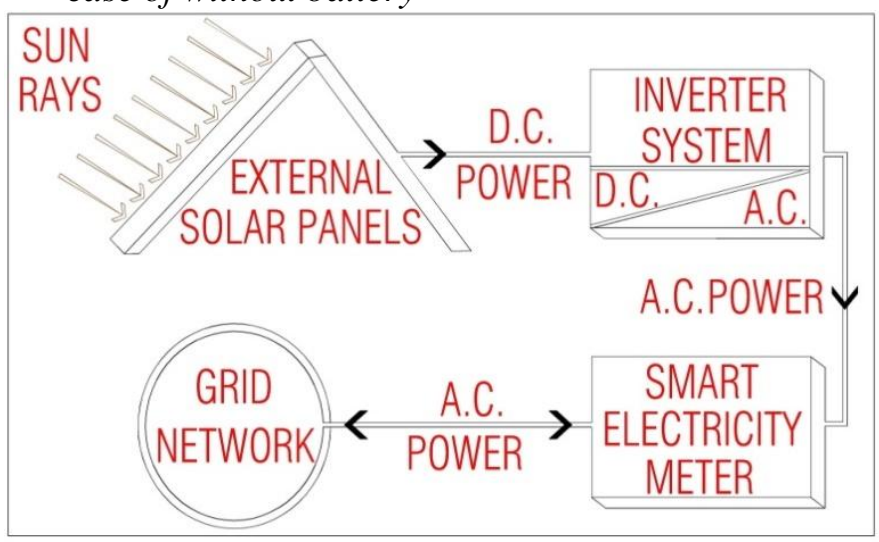

Fig 5: Current flow in components of PV system without battery

\subsubsection{Current flow in components of PV system in case of with battery}




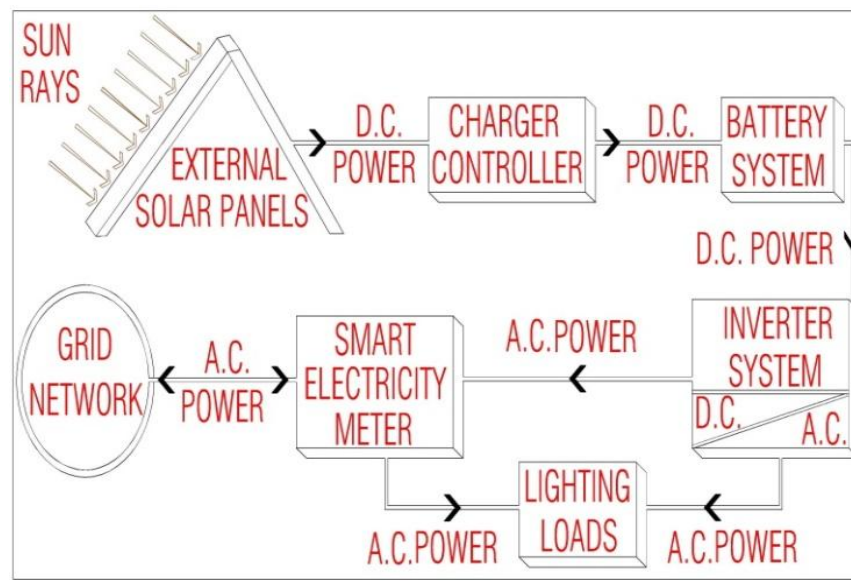

Fig 6: Current flow in components of PV system with battery

\section{RESULTS}

For $\varphi=25^{\circ}$ (Dubai, UAE), Tilt angle $\beta=30^{\circ}$. Monthly daily average energy produced by $90 \mathrm{kWp}$ array installed in Dubai $\left(25^{\circ} \mathrm{N}\right)$ in $\mathrm{kWh}$ per day as shown below:-

\subsection{Case 1 (Without Battery)}

4.1.1 For tilt angle $\left(\beta=0^{\circ}\right)$ :

Table 2. Monthly daily average energy generated and delivered by $90 \mathrm{kWp}$ at $\left(\beta=0^{\circ}\right)$ in case of without battery

\begin{tabular}{|c|c|c|c|c|c|c|}
\hline Month & Jan & Feb & Mar & Apr & May & Jun \\
\hline $\begin{array}{c}\text { Energy } \\
\text { generate } \\
\text { d } \\
\text { kWh/day }\end{array}$ & $\begin{array}{c}349 . \\
6\end{array}$ & 467.7 & 564.9 & 627.0 & 646.8 & 687.9 \\
\hline $\begin{array}{c}\text { Energy } \\
\text { delivered } \\
\text { kWh/day }\end{array}$ & $\begin{array}{c}312 . \\
9\end{array}$ & 430.4 & 528.2 & 591.4 & 612.2 & 653.2 \\
\hline Month & Jul & Aug & Sep & Oct & Nov & Dec \\
\hline $\begin{array}{c}\text { Energy } \\
\text { generate } \\
\text { d } \\
\text { kWh/day }\end{array}$ & 657. & 598.9 & 513.1 & 424.7 & 332.7 & 303.2 \\
\hline $\begin{array}{c}\text { Energy } \\
\text { delivered } \\
\text { kWh/day }\end{array}$ & 623. & 564.0 & 477.9 & 388.7 & 296.6 & 267.2 \\
\hline
\end{tabular}

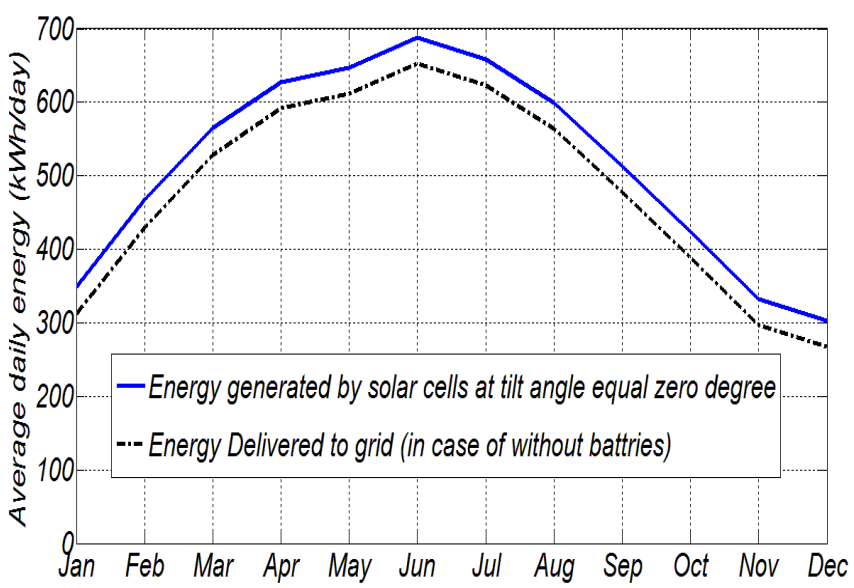

Fig 7: Monthly daily average energy by $90 \mathrm{kWp}$ in Dubai $\left(25^{\circ} \mathrm{N}\right)$ at $\left(\beta=0^{\circ}\right)$ in case of without battery

\subsubsection{For tilt angle $\left(\beta=15^{\circ}\right)$.}

Table 3. Monthly daily average energy generated and delivered by $90 \mathrm{kWp}$ at $\left(\beta=15^{\circ}\right)$ in case of without battery

\begin{tabular}{|c|c|c|c|c|c|c|}
\hline Month & Jan & Feb & Mar & Apr & May & Jun \\
\hline $\begin{array}{c}\text { Energy } \\
\text { generate } \\
\text { d } \\
\text { kWh/day }\end{array}$ & $\begin{array}{c}545 . \\
8\end{array}$ & 639.8 & 666.1 & 642.6 & 593.9 & 599.8 \\
\hline $\begin{array}{c}\text { Energy } \\
\text { delivered } \\
\text { kWh/day }\end{array}$ & $\begin{array}{c}509 . \\
1\end{array}$ & 602.4 & 629.3 & 607.0 & 559.3 & 565.1 \\
\hline Month & Jul & Aug & Sep & Oct & Nov & Dec \\
\hline $\begin{array}{c}\text { Energy } \\
\text { generate } \\
\text { d } \\
\text { kWh/day }\end{array}$ & $\begin{array}{c}585 . \\
6\end{array}$ & 587.4 & 583.4 & 572.9 & 528.6 & 509.4 \\
\hline $\begin{array}{c}\text { Energy } \\
\text { delivered } \\
\text { kWh/day }\end{array}$ & $\begin{array}{c}551 . \\
0\end{array}$ & 552.5 & 548.2 & 536.9 & 492.5 & 473.4 \\
\hline
\end{tabular}

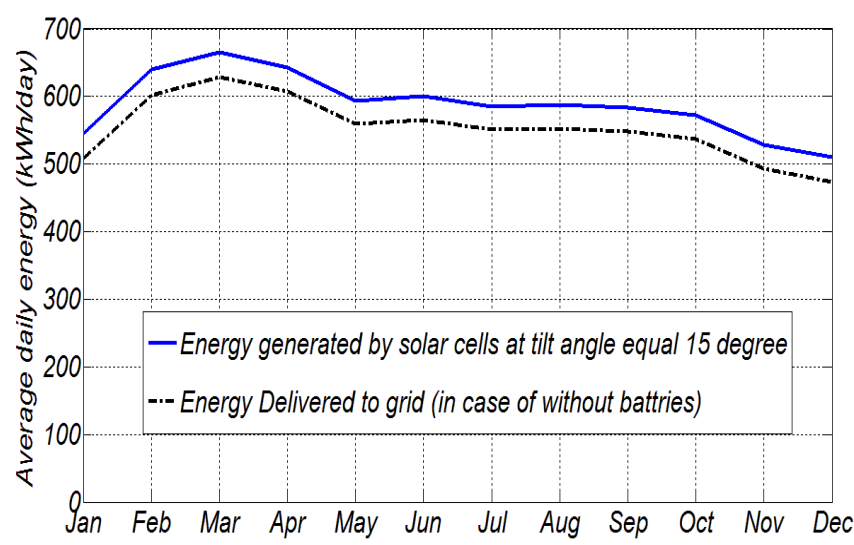

Fig 8: Monthly daily average energy by $90 \mathrm{kWp}$ in Dubai $\left(25^{\circ} \mathrm{N}\right)$ at $\left(\beta=15^{\circ}\right)$ in case of without battery 


\subsubsection{For tilt angle $\left(\beta=30^{\circ}\right)$ :}

Table 4. Monthly daily average energy generated and delivered by $90 \mathrm{kWp}$ at $\left(\beta=30^{\circ}\right)$ in case of without battery

\begin{tabular}{|c|c|c|c|c|c|c|}
\hline Month & Jan & Feb & Mar & Apr & May & Jun \\
\hline $\begin{array}{c}\text { Energy } \\
\text { generate } \\
\text { d } \\
\text { kWh/day }\end{array}$ & $\begin{array}{c}703 . \\
2\end{array}$ & 761.1 & 710.1 & 602.7 & 492.0 & 466.1 \\
\hline $\begin{array}{c}\text { Energy } \\
\text { delivered } \\
\text { kWh/day }\end{array}$ & $\begin{array}{c}666 . \\
\text { Month }\end{array}$ & 723.7 & 673.3 & 567.1 & 457.5 & 431.4 \\
\hline $\begin{array}{c}\text { Energy } \\
\text { generate } \\
\text { d } \\
\text { kWh/day }\end{array}$ & $\begin{array}{c}465 . \\
5\end{array}$ & 521.0 & 596.8 & 668.3 & 682.2 & 680.3 \\
\hline $\begin{array}{c}\text { Energy } \\
\text { delivered } \\
\text { kWh/day }\end{array}$ & $\begin{array}{c}430 . \\
9\end{array}$ & 486.1 & 561.6 & 632.3 & 646.2 & 644.2 \\
\hline
\end{tabular}

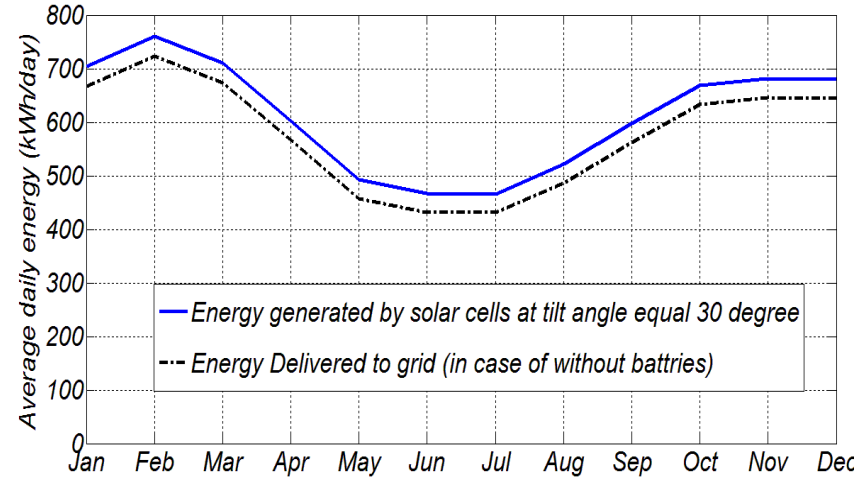

Fig 9: Monthly daily average energy by $90 \mathrm{kWp}$ in Dubai $\left(25^{\circ} \mathrm{N}\right)$ at $\left(\beta=30^{\circ}\right)$ in case of without battery

4.1.4 For tilt angle $\left(\beta=45^{\circ}\right)$ :

Table 5. Monthly daily average energy generated and delivered by $90 \mathrm{kWp}$ at $\left(\beta=45^{\circ}\right)$ in case of without battery

\begin{tabular}{|c|c|c|c|c|c|c|}
\hline Month & Jan & Feb & Mar & Apr & May & Jun \\
\hline $\begin{array}{c}\text { Energy } \\
\text { generate } \\
\text { d } \\
\text { kWh/day } \\
\end{array}$ & $\begin{array}{c}811 . \\
3\end{array}$ & 823.2 & 694.0 & 509.9 & 348.1 & 295.5 \\
\hline $\begin{array}{c}\text { Energy } \\
\text { delivered } \\
\text { kWh/day }\end{array}$ & $\begin{array}{c}774 . \\
5\end{array}$ & 785.9 & 657.2 & 474.3 & 313.5 & 260.8 \\
\hline Month & Jul & Aug & Sep & Oct & Nov & Dec \\
\hline $\begin{array}{c}\text { Energy } \\
\text { generate } \\
\text { d } \\
\text { kWh/day }\end{array}$ & $\begin{array}{c}305 . \\
5\end{array}$ & 404.2 & 552.5 & 704.2 & 783.0 & 803.8 \\
\hline $\begin{array}{c}\text { Energy } \\
\text { delivered } \\
\text { kWh/day }\end{array}$ & $\begin{array}{c}270 . \\
9\end{array}$ & 369.3 & 517.3 & 668.2 & 746.9 & 767.8 \\
\hline
\end{tabular}

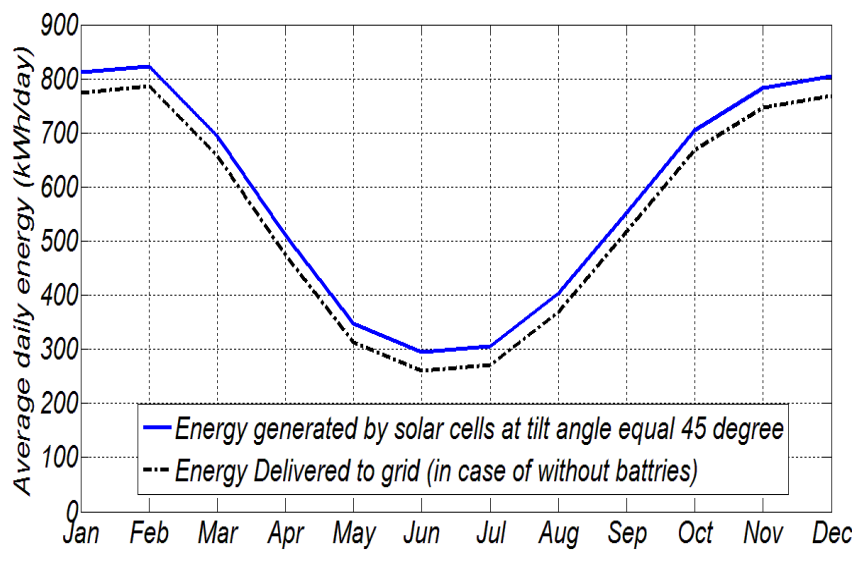

Fig 10: Monthly daily average energy by $90 \mathrm{kWp}$ in Dubai $\left(25^{\circ} \mathrm{N}\right)$ at $\left(\beta=45^{\circ}\right)$ in case of without battery

\subsection{Case 2 (With the Battery)}

4.2.1 For tilt angle $\left(\beta=0^{\circ}\right)$ :

Table 6. Monthly daily average energy generated and delivered by $90 \mathrm{kWp}$ at $\left(\beta=0^{\circ}\right)$ in case of with the battery

\begin{tabular}{|c|c|c|c|c|c|c|}
\hline Month & Jan & Feb & Mar & Apr & May & Jun \\
\hline $\begin{array}{c}\text { Energy } \\
\text { generate } \\
\text { d } \\
\text { kWh/day }\end{array}$ & $\begin{array}{c}349 . \\
6\end{array}$ & 467.7 & 564.9 & 627.0 & 646.8 & 687.9 \\
\hline $\begin{array}{c}\text { Energy } \\
\text { delivered } \\
\text { kWh/day }\end{array}$ & $\begin{array}{c}307 . \\
0\end{array}$ & 421.5 & 515.8 & 576.2 & 595.2 & 634.6 \\
\hline Month & Jul & Aug & Sep & Oct & Nov & Dec \\
\hline $\begin{array}{c}\text { Energy } \\
\text { generate } \\
\text { d } \\
\text { kWh/day }\end{array}$ & $\begin{array}{c}657 . \\
7\end{array}$ & 598.9 & 513.1 & 424.7 & 332.7 & 303.2 \\
\hline $\begin{array}{c}\text { Energy } \\
\text { delivered } \\
\text { kWh/day }\end{array}$ & 605. & 549.5 & 466.6 & 380.7 & 291.3 & 262.3 \\
\hline
\end{tabular}

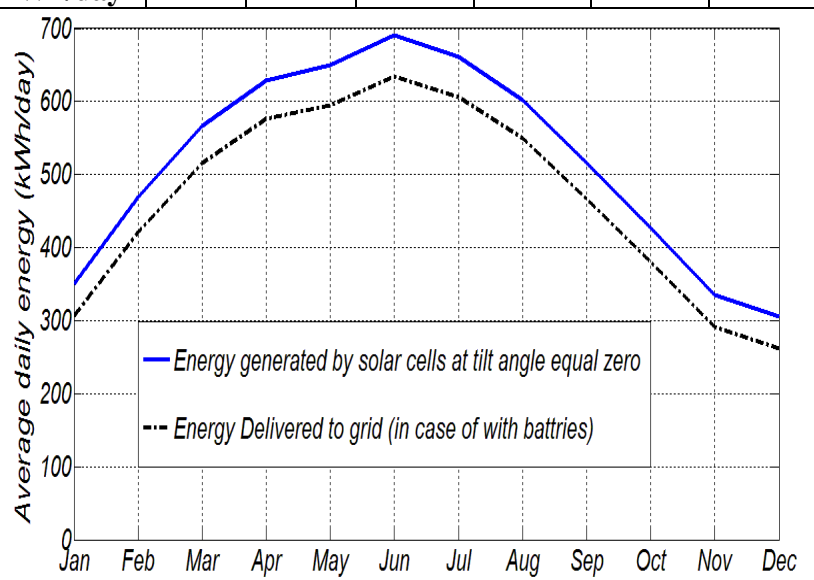

Fig 11: Monthly daily average energy by $90 \mathrm{kWp}$ in Dubai $\left(25^{\circ} \mathrm{N}\right)$ at $\left(\beta=0^{\circ}\right)$ in case of with battery 


\subsubsection{For tilt angle $\left(\beta=15^{\circ}\right)$ :}

Table 7 . Monthly daily average energy generated and delivered by $90 \mathrm{kWp}$ at $\left(\beta=15^{\circ}\right)$ in case of with the battery

\begin{tabular}{|c|c|c|c|c|c|c|}
\hline Month & Jan & Feb & Mar & Apr & May & Jun \\
\hline $\begin{array}{c}\text { Energy } \\
\text { generate } \\
\text { d } \\
\text { kWh/day }\end{array}$ & $\begin{array}{c}545 . \\
8\end{array}$ & 639.8 & 666.1 & 642.6 & 593.9 & 599.8 \\
\hline $\begin{array}{c}\text { Energy } \\
\text { delivered } \\
\text { kWh/day }\end{array}$ & $\begin{array}{c}498 . \\
2\end{array}$ & 589.8 & 615.6 & 592.9 & 545.1 & 550.7 \\
\hline Month & Jul & Aug & Sep & Oct & Nov & Dec \\
\hline $\begin{array}{c}\text { Energy } \\
\text { generate } \\
\text { d } \\
\text { kWh/day }\end{array}$ & $\begin{array}{c}585 . \\
6\end{array}$ & 587.4 & 583.4 & 572.9 & 528.6 & 509.4 \\
\hline $\begin{array}{c}\text { Energy } \\
\text { delivered } \\
\text { kWh/day }\end{array}$ & 536. & 539.7 & 536.4 & 526.2 & 482.9 & 463.3 \\
\hline
\end{tabular}

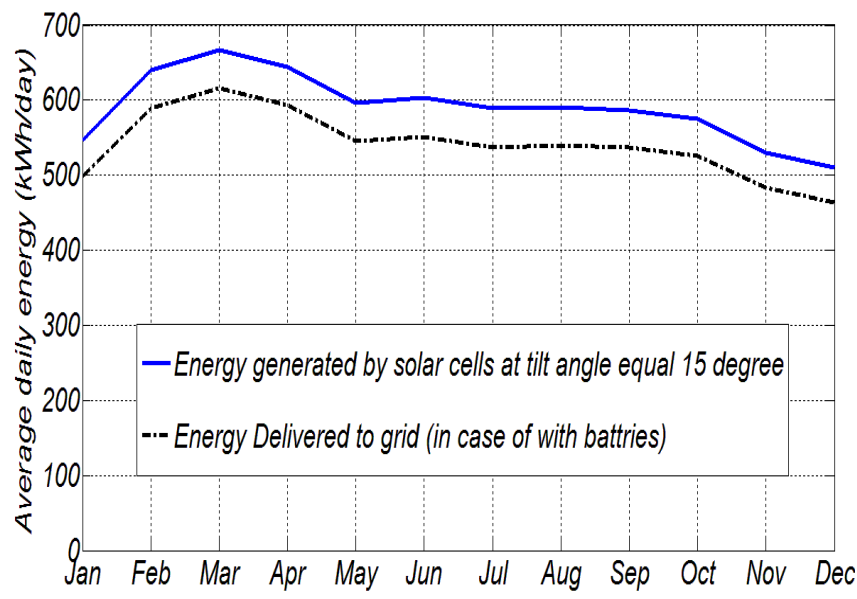

Fig 12: Monthly daily average energy by $90 \mathrm{kWp}$ in Dubai $\left(25^{\circ} \mathrm{N}\right)$ at $\left(\beta=15^{\circ}\right)$ in case of with the battery

\subsubsection{For tilt angle $\left(\beta=30^{\circ}\right)$.}

Table 8. Monthly daily average energy generated and delivered by $90 \mathrm{kWp}$ at $\left(\beta=30^{\circ}\right)$ in case of with the battery

\begin{tabular}{|c|c|c|c|c|c|c|}
\hline Month & Jan & Feb & Mar & Apr & May & Jun \\
\hline $\begin{array}{c}\text { Energy } \\
\text { generate } \\
\text { d } \\
\text { kWh/day }\end{array}$ & $\begin{array}{c}703 . \\
2\end{array}$ & 761.1 & 710.1 & 602.7 & 492.0 & 466.1 \\
\hline $\begin{array}{c}\text { Energy } \\
\text { delivered } \\
\text { kWh/day }\end{array}$ & $\begin{array}{c}651 . \\
6\end{array}$ & 708.2 & 658.8 & 545.5 & 446.5 & 420.7 \\
\hline Month & Jul & Aug & Sep & Oct & Nov & Dec \\
\hline $\begin{array}{c}\text { Energy } \\
\text { generate } \\
\text { d } \\
\text { kWh/day } \\
\end{array}$ & $\begin{array}{c}465 . \\
5\end{array}$ & 521.0 & 596.8 & 668.3 & 682.2 & 680.3 \\
\hline $\begin{array}{c}\text { Energy } \\
\text { delivered } \\
\text { kWh/day }\end{array}$ & $\begin{array}{c}420 . \\
5\end{array}$ & 475.5 & 550.1 & 619.7 & 632.7 & 629.9 \\
\hline
\end{tabular}

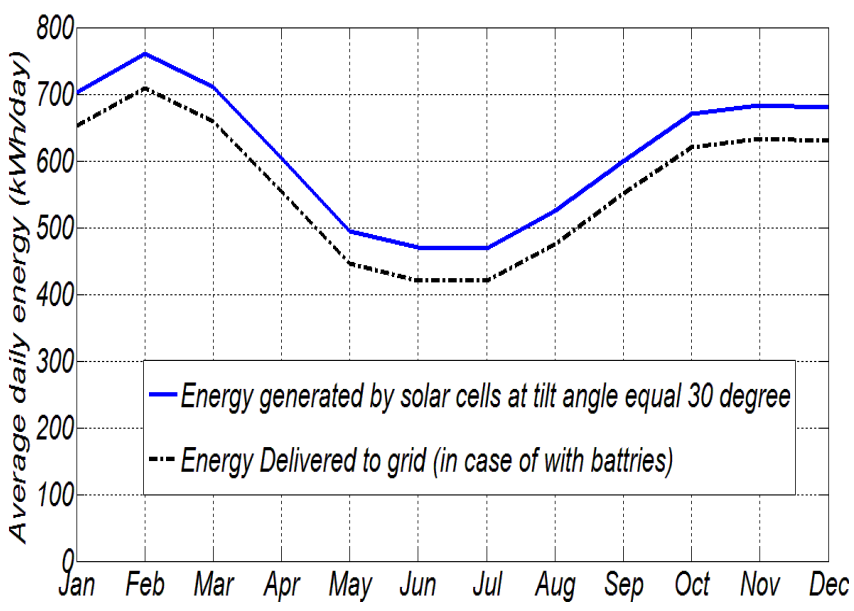

Fig 13: Monthly daily average energy by $90 \mathrm{kWp}$ in Dubai $\left(25^{\circ} \mathrm{N}\right)$ at $\left(\beta=30^{\circ}\right)$ in case of with the battery

4.2.4 For tilt angle $\left(\beta=45^{\circ}\right)$ :

Table 9. Monthly daily average energy generated and delivered by $90 \mathrm{kWp}$ at $\left(\beta=45^{\circ}\right)$ in case of with the battery

\begin{tabular}{|c|c|c|c|c|c|c|}
\hline Month & Jan & Feb & Mar & Apr & May & Jun \\
\hline $\begin{array}{c}\text { Energy } \\
\text { generate } \\
\text { d } \\
\text { kWh/day }\end{array}$ & $\begin{array}{c}811 . \\
3\end{array}$ & 823.2 & 694.0 & 509.9 & 348.1 & 295.5 \\
\hline $\begin{array}{c}\text { Energy } \\
\text { delivered } \\
\text { kWh/day }\end{array}$ & $\begin{array}{c}755 . \\
7\end{array}$ & 768.0 & 642.7 & 463.8 & 306.2 & 254.8 \\
\hline Month & Jul & Aug & Sep & Oct & Nov & Dec \\
\hline $\begin{array}{c}\text { Energy } \\
\text { generate } \\
\text { d } \\
\text { kWh/day }\end{array}$ & $\begin{array}{c}305 . \\
5\end{array}$ & 404.2 & 552.5 & 704.2 & 783.0 & 803.8 \\
\hline $\begin{array}{c}\text { Energy } \\
\text { delivered } \\
\text { kWh/day }\end{array}$ & $\begin{array}{c}264 . \\
6\end{array}$ & 361.3 & 506.4 & 654.1 & 730.3 & 748.9 \\
\hline
\end{tabular}

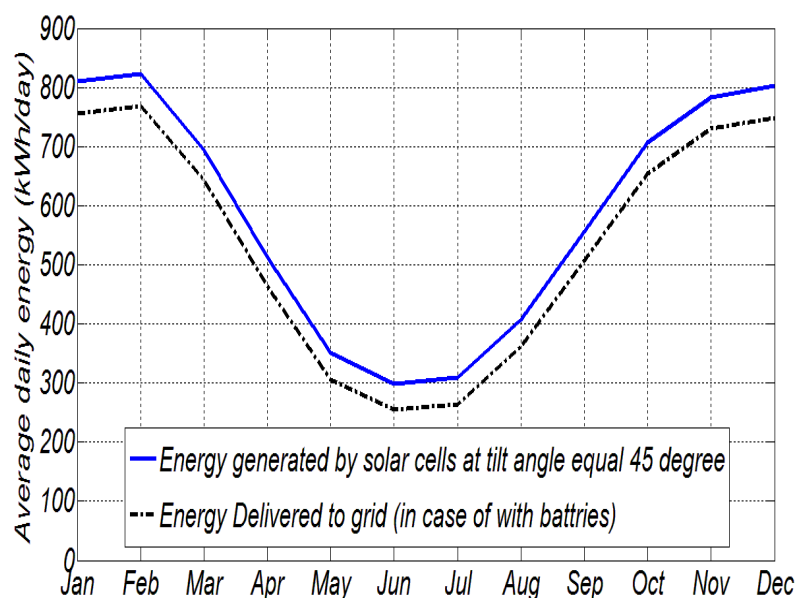

Fig 14: Monthly daily average energy by $90 \mathrm{kWp}$ in Dubai $\left(25^{\circ} \mathrm{N}\right)$ at $\left(\beta=45^{\circ}\right)$ in case of with the battery 


\subsection{Compare Results}

Table 10. Monthly daily average energy generated vs. variation of tilt angle $(\beta)$ by $90 \mathrm{kWp}$

$$
P=\frac{A}{1+i}+\frac{A}{(1+i)^{2}}+\ldots+\frac{A}{(1+i)^{N}}
$$

\begin{tabular}{|c|c|c|c|c|c|}
\hline $\begin{array}{c}\text { Tilt angle } \\
\text { (degree) }\end{array}$ & $\mathbf{0}$ & $\mathbf{5}$ & $\mathbf{1 0}$ & $\mathbf{1 5}$ & $\mathbf{2 0}$ \\
\hline $\begin{array}{c}\text { Energy delivered } \\
\text { MWh/year } \\
\text { (Without } \\
\text { Battery) }\end{array}$ & $\begin{array}{c}170 . \\
6\end{array}$ & $\begin{array}{c}181 . \\
2\end{array}$ & 190.1 & 197.1 & 202.0 \\
\hline $\begin{array}{c}\text { Energy delivered } \\
\text { MWh/year } \\
\text { (With Battery) }\end{array}$ & $\begin{array}{c}174 . \\
8\end{array}$ & $\begin{array}{c}185 . \\
6\end{array}$ & 194.6 & 201.6 & 206.6 \\
\hline $\begin{array}{c}\text { Tilt angle } \\
\text { (degree) }\end{array}$ & $\mathbf{3 0}$ & $\mathbf{3 5}$ & $\mathbf{4 0}$ & $\mathbf{4 5}$ & $\mathbf{5 0}$ \\
\hline $\begin{array}{c}\text { Energy delivered } \\
\text { MWh/year } \\
\text { (Without } \\
\text { Battery) }\end{array}$ & $\begin{array}{c}205 . \\
9\end{array}$ & $\begin{array}{c}204 . \\
8\end{array}$ & 201.6 & 196.4 & 189.3 \\
\hline $\begin{array}{c}\text { Energy delivered } \\
\text { MWh/year } \\
\text { (With Battery) }\end{array}$ & $\begin{array}{c}210 . \\
5\end{array}$ & $\begin{array}{c}209 . \\
4\end{array}$ & 206.2 & 210.0 & 193.8 \\
\hline
\end{tabular}

Where that: $(\mathrm{P})$ is the initial payment at starting moment, (A) is the installment of payment, (i) is the annual interest rate and $(\mathrm{N})$ is the number of Installments.

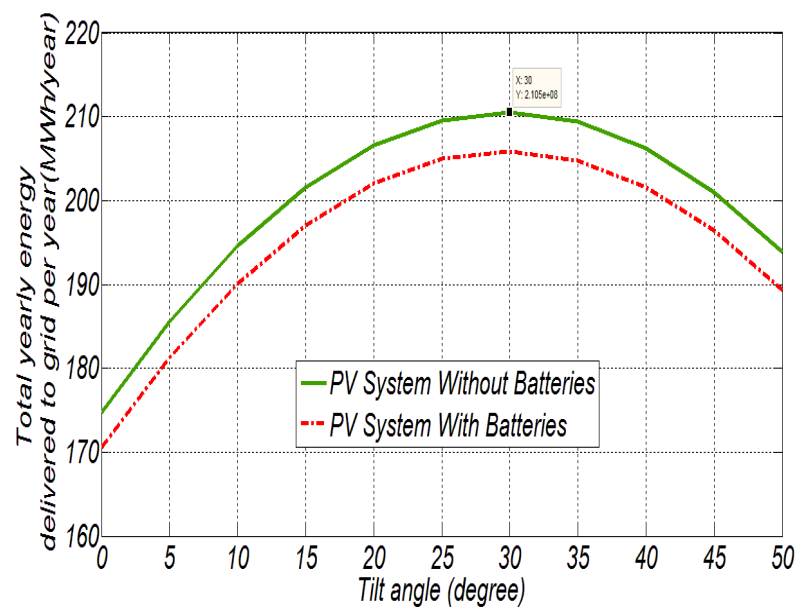

Fig 15: Monthly daily average energy generated vs. variation of tilt angle $(\beta)$ by $90 \mathrm{kWp}$ in Dubai $\left(25^{\circ} \mathrm{N}\right)$

As shown in both of the above Figure and Table, the maximum output energy yearly achieved at a tilt angle $(\beta$ $=30^{\circ}$ ). Moreover, the output energy yearly is more in case of without batteries than in case of with the batteries.

\section{ECONOMIC ANALAYSIS}

Note that:

- Interest rate $(\mathrm{i}=3 \%)$ as an annual loan.

- Average power for the site is $300 \mathrm{~kW}$.

- $\quad$ The system loss is $\left(\mathrm{L}_{\mathrm{S}}=2 \%\right)$.

\subsection{Economic Equations}

The system cost is $\mathrm{P}(\$)$ paid at $\mathrm{t}=0$, and this amount is borrowed from a bank and will return back by $\mathrm{N}$ equal annual payments for each A per year. The Eq. (12) appears the relation between $\mathrm{P}, \mathrm{A}, \mathrm{N}$ and interest rate $\mathrm{i}[12]$.

$$
P_{t}=E * C_{o}\left[\frac{1-\left(\frac{1-L_{s}}{1+i_{m}}\right)^{M}}{i_{m}-L_{s}}\right]
$$

Combining between Eq.'s (12) \& (13), we will get Eq. (14): $P=A *\left[\frac{1-(1+i)^{-N}}{i}\right]$

Where that: $(\mathrm{Pt})$ is the total system cost over 25 years, $(\mathrm{E})$ is the average monthly generated energy $(\mathrm{kWh}),\left(\mathrm{C}_{\mathrm{o}}\right)$ is the selling price per $\mathrm{kWh},\left(\mathrm{L}_{\mathrm{S}}\right)$ is the monthly loss in system efficiency, $\left(\mathrm{i}_{\mathrm{m}}\right)$ is the monthly interest rate and $(\mathrm{M})$ is the total number of months.

\subsection{International Market Prices}

Table 11. International market prices for each item in the

\begin{tabular}{|c|c|c|}
\hline Item & Cost & $\begin{array}{l}\text { Average } \\
\text { lifetime }\end{array}$ \\
\hline \multicolumn{2}{|l|}{ PV Module } & $\begin{array}{l}25 \text { years for } \\
\text { PV Module }\end{array}$ \\
\hline Modules cost per $\mathbf{k W p}$ & $700 \$ / \mathrm{kWp}$ & \\
\hline Mounting cost per $\mathrm{kWp}$ & $200 \$ / \mathrm{kWp}$ & \\
\hline Installation cost per $\mathrm{kWp}$ & $100 \$ / \mathrm{kWp}$ & \\
\hline PV system maintenance yearly & $1000 \$ /$ site & \\
\hline \multicolumn{2}{|l|}{$20 \mathrm{kWp}$ Inverter } & $\begin{array}{c}10 \text { years for } \\
\text { Inverter }\end{array}$ \\
\hline Inverter cost & $3700 \$$ & \\
\hline Mounting cost & $1000 \$$ & \\
\hline Installation cost & $550 \$$ & \\
\hline Cables per inverter & $170 \$$ & \\
\hline \multicolumn{2}{|l|}{ Battery } & $\begin{array}{c}3 \text { years for } \\
\text { Battery }\end{array}$ \\
\hline $\begin{array}{c}\text { Battery and Mounting } \\
\text { cost } / \mathbf{k W p}\end{array}$ & $110 \$$ & \\
\hline Installation cost & $5 \$$ & \\
\hline
\end{tabular}
PV system and it's average lifetime

\subsection{Cash Flow Diagram}




\subsubsection{Without battery}

The figure below shows the lifetime of PV system components. PV modules will not replace during the overall 25 years. Inverters should be replaced every ten years, which means twice overall 25 years. Furthermore, every year there are bills like electricity every month, maintenance of the system...etc., should be paid.

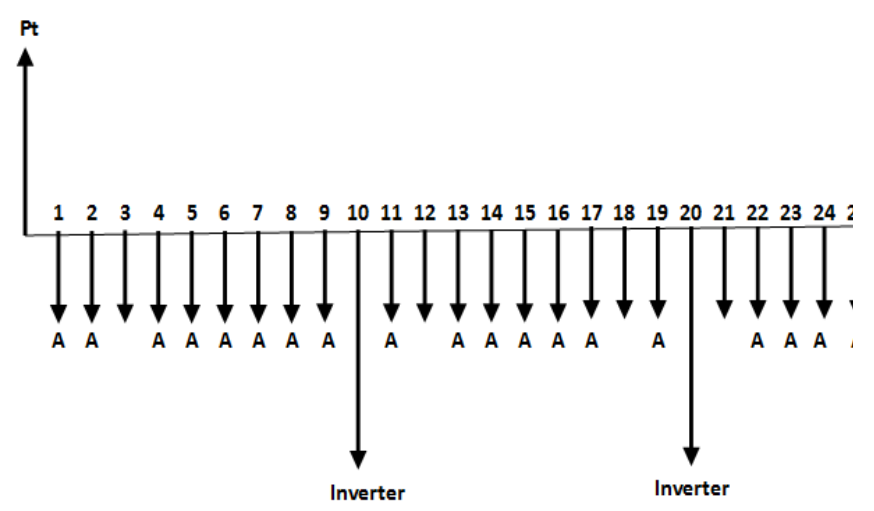

Fig 16: The cash flow diagram for without battery

\subsubsection{With the battery}

The same description like the figure above, but in this case (with batteries), batteries should be replaced every three years which means eight times overall 25 years.

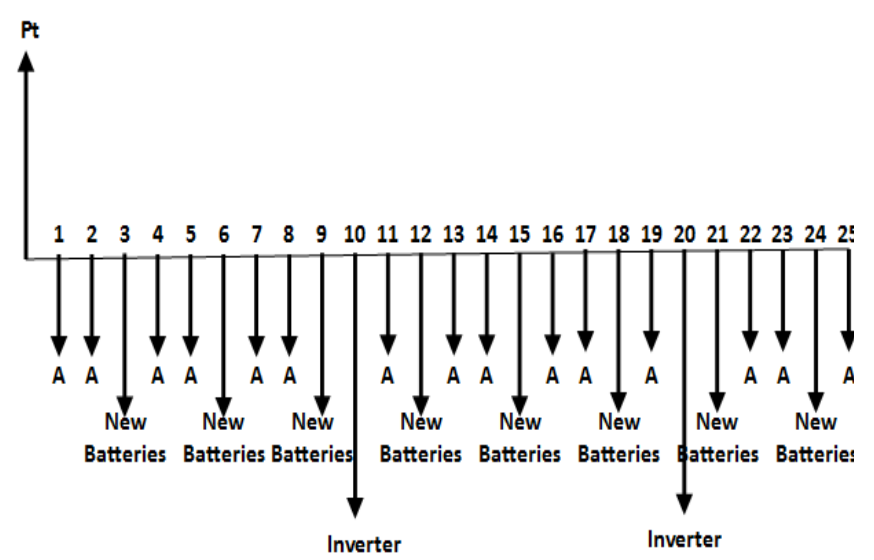

Fig 17: The cash flow diagram for the case of with the battery

\subsection{Economics Comparison Table}

Note that:

- All of the economic analysis calculated at $\left(\beta=30^{\circ}\right)$, which gives the maximum energy all over the year.

- The cost of $1 \mathrm{kWh}$ for the consumer is $0.104 \$$ in the UAE.

Table 12. Economics comparison table in both cases (without \& with) the battery

\begin{tabular}{|c|c|c|}
\hline Element & $\begin{array}{c}\text { Without } \\
\text { Battery }\end{array}$ & $\begin{array}{c}\text { With } \\
\text { Battery }\end{array}$ \\
\hline Average lighting energy (kW) & 37.5 & 50.08 \\
\hline PV array size (kWp) & \multicolumn{2}{|c|}{90} \\
\hline Battery size (Ah) & ------ & 1,500 \\
\hline Total PV modules cost (\$) & \multicolumn{2}{|c|}{90,000} \\
\hline Total inverters cost (\$) & \multicolumn{2}{|c|}{62,660} \\
\hline Total batteries cost (\$) & ------ & 128,288 \\
\hline
\end{tabular}

\begin{tabular}{|c|c|c|}
\hline $\begin{array}{c}\text { Total primitive maintenance cost } \\
\text { for PV (\$) }\end{array}$ & \multicolumn{2}{|c|}{17,642} \\
\hline $\begin{array}{c}\text { Total bill paid to grid PV system } \\
\text { overall 25 years }\end{array}$ & \multicolumn{2}{|c|}{210,276} \\
\hline $\begin{array}{c}\text { Overall 25 years total system cost } \\
\text { (\$) }\end{array}$ & 380,578 & 508,866 \\
\hline Cost of energy (\$/kWh) & 0.15 & 0.21 \\
\hline $\begin{array}{c}\text { Total energy delivered to grid } \\
\text { (MWh/year) }\end{array}$ & 210.52 & 205.89 \\
\hline
\end{tabular}

\section{CONCLUSION}

At the same time, if humanity is to reach population levels of 9.2 billion by 2050 , this level of population is unsustainable without cities. While city authorities are key players, they don't have all the answers, or all the powers, and certainly not the money. They have to plan both to combat climate change and to mitigate the effects of climate change, taking a view, in effect, on how successful or otherwise global efforts to reduce $\mathrm{Co}_{2}$ are likely to be. Here we can more believe in the importance of Sustainability. One of the fundamental sustainability problems today is in how we should use energy and materials. More sustainable pathways for sustainable energy systems will require step-wise changes in an evolutionary way, which might be difficult to fit into traditional long-term planning practice. Models for this kind of evolution can partly be found in the field of ecology. The consolidation of the thought of sustainability among all members of society, it has become very important to governments. Throughout applying of PV systems on different buildings in the country will be saving more fuels and costs of generation and transportation of the electricity which increase day after day. Where that we can save approximately $300 \mathrm{~kW}$ day of each residential building like the design of the villa in this paper, it will save 300 liter from refined fuel for this building only. Which will save approximately $600 \$$ and this value without adding costs of the prospecting processing, the refining of fossil fuel, the transportation and the distribution. So, applying of sustainability criteria is the best solution for the energy crisis which growing globally.

\section{REFERENCES}

[1] U.S. Energy Information Administration. May 2016, "International Energy Outlook 2016", https://www.eia.gov/.

[2] Conserve Energy Future. 2016,"Pros and Cons of Fossil Fuels", http://www.conserve-energyfuture.com/advantages-and-disadvantages-of-fossilfuels.php.

[3] Ahmed Naobei. 2008,"Pros of Nuclear Power", Nuclear Physics, Committed Copyright Dar Arab Thought.

[4] Hani Saied. 2014,"Cons of Nuclear Power”, Arageek eBook, www.arageek.comm.

[5] Conserve Energy Future. 2016,"Pros and Cons of Renewable Energy", http://www.conserve-energyfuture.com/advantages-and-disadvantages-of-renewableenergy.php.

[6] University of Alberta Office of Sustainability. 2014,"What is Sustainability?", http://sustainability.ualberta.ca/. 
[7] World Bank. 2016,"Electric power consumption (kWh per capita) in UAE", IEA Statistics (C) OECD/IEA, http://www.worldbank.org/.

[8] Invest in Group. Aug 2016,"10 Things to Know about the UAE's Renewable Energy Landscape", http://investingroup.org/.

[9] Wagdy R. Anis. 1985,"Design of Photovoltaic Systems for Tropical Climates", Ph.D thesis, Dept. of Electrical Eng., Catholic Univ. of Loavain, Belgium.

[10] Klein and S. A. 1977, “Calculation of Monthly Average Insolation on Tilted Surfaces”, Solar Energy, 19(4), 325329.

[11] Watmuff, J. H., Charters, W. W. S., and Proctor D. 1977, "Solar and Wind Induced External Coefficients Solar Collectors", Cooperation Mediterraneenne Pour 'Energie Solaire, 1, 56.

[12] Blank L. and Tarquin A. 2005,"McGraw-Hill Series in Industrial Engineering and Management Science", Engineering Economy.
[13] Ziyi Wang, Qinxing Wang, Ronald Wennersten and Qie Sun. 2015,"Mc Transitions to sustainable energy and material systems-outline of principles for scenarios", Energy Procedia vol. 75, pp. $2683-2693$.

[14] Javid Mohtasham. 2015,"Review Article-Renewable Energies", Energy Procedia vol. 74, pp. 1289 - 1297.

[15] Elizabeth May Kruger. 2015,'Options for Sustainability in Building and Energy: A South African Permaculture Case Study", Energy Procedia vol. 83, pp. 544 - 554.

[16] Hassan A. N. Hejase and Ali H. Assi. Oct 2013,"Global and Diffuse Solar Radiation in the United Arab Emirates", International Journal of Environmental Science and Development vol. 4, no. 5.

[17] Muhammed A. Ahmed and Sidra A. Shaikh. 2013,"Solar Radiation Studies for Dubai and Sharjah, UAE", International Journal of Mathematical, Computational, Physical, Electrical and Computer Engineering vol. 7, no. 1. 